\title{
Control of Aberration and Space-Charge Effects in the Orbitrap Mass Analyzer
}

\author{
Dmitry Grinfeld ${ }^{1}$, Mikhail Monastyrskiy ${ }^{2}$, and Alexander Makarov ${ }^{1}$ \\ 1. Thermo Fisher Scientific, Bremen, Germany \\ 2. Prokhorov General Physics Institute, Rus. Acad. Sci., Moscow, Russia
}

The idea of orbital ion confinement dates back to 1923 when the orbital ion trap was proposed and implemented by Kingdon [1]. In the following several decades this principle was often used in ion spectroscopy but it was not until 1981 that Knight proposed a mass-selective orbital ion trap [2]. Similar to Kingdon trap, the ions were trapped revolving around a negatively biased wire. The ions generated by laser ionization were captured in the trap where rudimentary mass analysis was performed by means of resonance excitation of axial oscillations between two conical guarding electrodes that formed a quasiharmonic potential well. This first attempt demonstrated that the quality of mass analysis mostly depends on the trapping field accuracy, and particularly, on the isochronism of the axial oscillatory motion.

The simplest axisymmetric electrostatic potential distribution with ideal isochronism in the axial direction $z$ is given by the formula

$$
\varphi_{0}(z, r)=V_{c} \frac{k_{0}}{2}\left(z^{2}-\frac{r^{2}}{2}+r_{m}^{2} \ln \frac{r}{r_{m}}\right)+\text { const }
$$

where $r_{m}$ and $\mathrm{k}_{0}$ are geometrical parameters and $V_{c}$ is the applied voltage. A detailed description of the ion dynamics in the quadro-logarithmic potential Eq. (1) can be found in the paper by Gall et al. [3]. The most important property of this unique potential distribution is that the motion equations are separable for the axial coordinate $z$ and the other coordinates (radius $r$ and the rotational angle $\psi$ ), and the axial motion is harmonic. The axial oscillation frequency in the ideal field is

$$
\omega=\sqrt{k_{0} V_{c}(q / m)}
$$

and thus depends on the ion's mass-to-charge ratio $m / q$ but not on the oscillation amplitude $Z$ and the revolving radius. It means that a bunch of identical ions, once injected into the trap, keeps oscillating as a single packet preserving the phase coherency. Having detected the oscillation frequency, one can determine the mass-to-charge ratio of the ions and construct a mass spectrum of the analyte. The other important property of the quadro-logarithmic potential is the confinement in the radial pseudopotential

$$
U(r)=\frac{\omega^{2}}{2}\left(r_{m}^{2} \ln \frac{r}{r_{m}}-\frac{r^{2}}{2}\right)+\frac{K^{2}}{2 m^{2} r^{2}},
$$

with $K$ being the conserved rotational momentum. This pseudopotential possesses a distinct minimum at

$$
r=r_{c}=\left(\frac{r_{m}^{2}}{2}-\sqrt{\frac{r_{m}^{4}}{4}-\frac{2 q K^{2}}{k_{0} m V_{c}}}\right)^{1 / 2}
$$


A stable orbit is restricted in-between the minimal and maximal radii $r^{\text {min }}$ and $r^{\text {max }}$ determined by the conserved radial full energy $U_{0}$ through the equation $U\left(r^{\min }\right)=U\left(r^{\max }\right)=U_{0}$. One can find a detailed analysis in $[3,4]$.

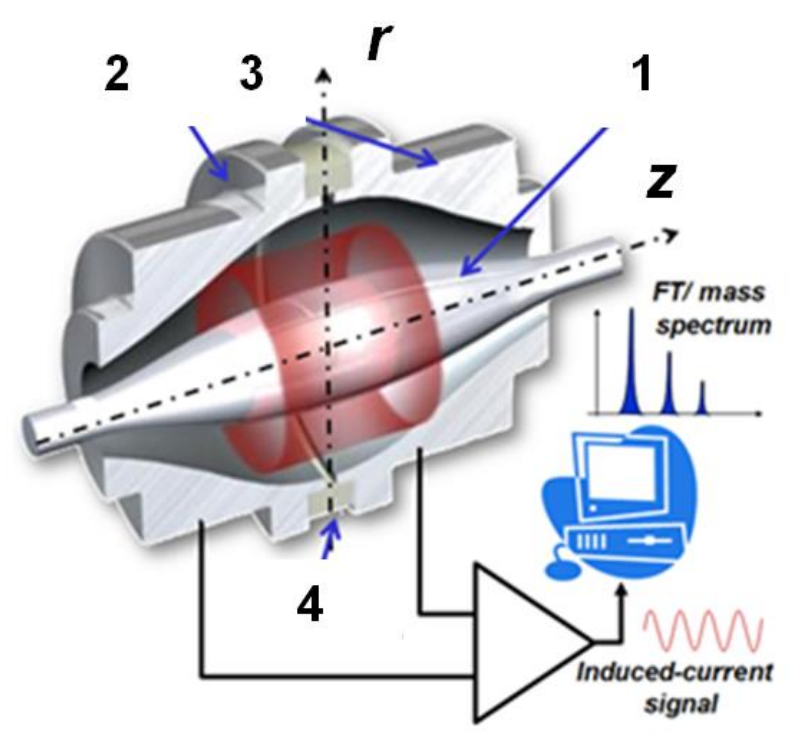

Figure 1. Design of the Orbitrap mass analyzer. 1 - central electrode $\left(\varphi=-V_{c}\right), 2,3$ - two halves of the outer electrode $(\varphi \approx 0)$ isolated by a quartz ring 4 . The induced current is amplified and digitally processed to obtain a mass spectrum of the trapped ions.

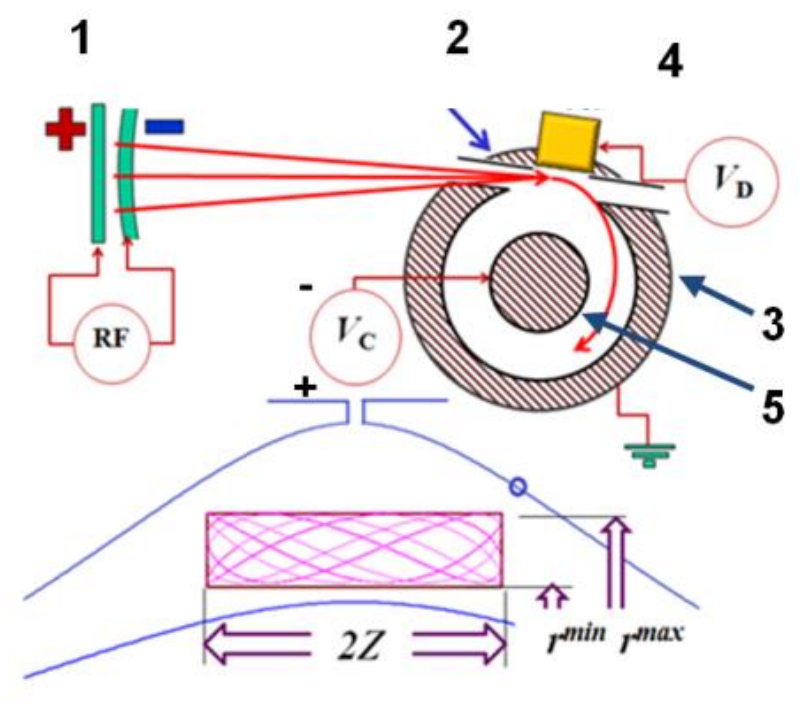

Figure 2. Ion injection scheme. Ions are stored and cooled in a gas-filled Paul trap 1 and then transversally ejected towards the injection slot 2 in the outer electrode 3 . The retarding voltage $V_{D}$ on the deflection electrode 4 and the attracting potential $-V_{c}$ of the central electrode 5 guide the ions into an orbit.

For practical implementation, the field according to Eq. (1) is generated between two spindle-like electrodes manufactured to mimic two equipotential surfaces as shown in Figure 1. Ions are injected tangentially through a narrow slot in the outer electrode whilst the central electrode voltage is gradually decreasing until it reaches the nominal value $-V_{c}$. This allows adiabatic radial squeezing of the ion orbits, so that the injected ions are safely separated from the outer electrode. Once injected, the ions remain trapped for a long time that is only restricted by residual gas collisions. With a typical vacuum $10^{-10}$ Torr, the trapping time is as long as several seconds [5].

For the image current detection, the outer electrode is split into two halves which are electrically isolated with a quartz ring as shown in Figure 1. The halves are maintained at virtually zero potential up to the induced voltage. The induced voltage signal is amplified, digitalized, and Fourier transformed to produce a spectrum of ion oscillatory frequencies $\omega$ and the distribution of ionic species in $m / q$. The mass resolving power $R=m / \Delta m$, where $\Delta m$ is the minimally resolved mass difference, is mostly determined by the signal acquisition time $T$ and the oscillation frequency as $R=T \omega / 2 \pi$. Provided that the frequency $\omega / 2 \pi \geq 10^{5} \mathrm{~Hz}$ (in the $\mathrm{m} / q$ range below $1000 \mathrm{Th}$ ), the limiting mass resolving power can reach several hundreds of thousands. 
If the signal acquisition time is not restricted by collisions or technical reasons, the signal duration and, therefore, the Orbitrap mass analyzer's resolving power, are limited by imperfection of the field, which makes the oscillation frequency $\omega$ deviate from its ideal value Eq. (2). In case that the frequency acquires dependence on the orbital parameters $Z, r^{\min }$, and $r^{\max }$, identical ions trapped on different orbits acquire slightly different frequencies with some dispersion $\delta \omega$. The frequency spread causes the ions' oscillation phases to randomize after $T=2 \pi / \delta \omega$ that is the actual acquisition time limit, after which the induced current can no longer be picked up. Consider the perturbed potential

$$
\varphi_{0}(z, r)+\delta \varphi(z, r, \psi, t)
$$

where the perturbation $\delta \varphi$ depends on the coordinates $z$ and $r$, as well as the rotational angle $\psi$ and the time $t$. The field perturbation is mainly caused by the injection slot in the outer electrode, manufacturing and assembly imperfections, and Coulomb interactions between the ions. The perturbation is generally as small as $\delta \varphi / V_{c} \lesssim 10^{-3}$. As we are committed to the high mass resolving power in the range of $R=10^{5} \ldots 10^{6}$, so that at least the first order perturbation with respect to $\delta \varphi / V_{c}$ must be taken into account and, if possible, compensated.

Ion motion in the perturbed potential Eq. (5) can be effectively decomposed into ideal motion and the relevant perturbation with the use of the substitution

$$
z=Z(t) \cos (\omega t+\zeta(t)), \quad d z / d t=-\omega Z(t) \sin (\omega t+\zeta(t))
$$

where $Z(t)$ is the oscillation amplitude, $\omega$ is the unperturbed frequency, and $\zeta(t)$ is the phase offset. It should be noted that even in the ideal field the frequencies of rotation and radial oscillations are not constant, which means that the ion cloud becomes randomized over the 'rings' $(\psi, r) \in[0 . .2 \pi] \times$ $\left[r^{\min } . . r^{\max }\right]$ after a small number $\sim 100$ of axial oscillations. The averaged ion density is proportional to the time $\Delta t=\Delta r / \dot{r}$ an ion spends within between $r$ and $r+\Delta r$ and is given by the formula $\rho(r)=$ $\rho_{0} / \sqrt{U_{0}-U(r)}$, where $U_{0}=U\left(r^{\min }\right)=U\left(r^{\max }\right)$ is the conserved radial full energy and $\rho_{0}$ is a normalization constant. Accordingly, the perturbation potential can be averaged with weight

$$
\delta \tilde{\varphi}\left(z, t, r^{\min }, r^{\max }\right)=\int_{0}^{2 \pi} d \psi \int_{r_{\min }}^{r_{\max }} d r \frac{\rho_{0}}{\sqrt{U_{0}-U(r)}} \delta \varphi(z, r, \psi, t),
$$

where a pair of more intuitive orbital parameters $r^{\min }$ and $r^{\max }$ is used instead of the motion integrals $K$ and $U_{0}$. The averaged perturbation determines evolution of the oscillation amplitude and phase:

$$
\frac{d Z}{d t}=\frac{q}{\omega m} \frac{\partial \delta \tilde{\varphi}}{\partial z} \sin (\omega t+\zeta), \quad \frac{d \zeta}{d t}=\frac{q}{\omega m Z} \frac{\partial \delta \tilde{\varphi}}{\partial z} \cos (\omega t+\zeta)
$$

Further we introduce the averaging technique for an approximate solution of these equations under the condition that the field perturbation leads to only small changes of the amplitude and phase during a single unperturbed period $\tau=2 \pi / \omega$. Nevertheless, the effect is not neglected over large time intervals, since the changes may accumulate in the course of many oscillations. Consider changes $\Delta Z=$ $Z(t+\tau)-Z(t)$ and $\Delta \zeta=\zeta(t+\tau)-\zeta(t)$ on a single period, and assuming these changes to be small, we introduce the averaged derivatives $\dot{Z}=\Delta Z / \tau$ and $\dot{\zeta}=\Delta \zeta / \tau$. Integration of the right-hand parts of 
Eq. (8) over a period gives equations

$$
\dot{Z}=\frac{q}{2 \pi \omega m} \int_{0}^{2 \pi} \delta \tilde{\varphi}_{Z}^{\prime}(Z \cos \chi, t, \ldots) \sin \chi d \chi, \quad \dot{\zeta}=\frac{q}{2 \pi \omega m Z} \int_{0}^{2 \pi} \delta \tilde{\varphi}_{z}^{\prime}(Z \cos \chi, t, \ldots) \cos \chi d \chi
$$

We will consider the case that the field perturbation results from the electrode imperfections and is thus static. Analysis of the Coulomb perturbation, which is time-dependent, appears more complicated and some particular cases have been considered in [6]. With the static perturbation only, the first equation in Eq. (9) immediately gives $\dot{Z}=0$, which is simply a manifestation of full energy conservation. The second equation is not so trivial and gives the oscillation frequency perturbation as a function of the three conserved orbital parameters:

$$
\frac{\Delta \omega\left(Z, r^{\min }, r^{\max }\right)}{\omega}=\frac{\dot{\zeta}}{\omega}=\int_{r_{\min }}^{r_{\max }} \frac{\rho_{0}}{\sqrt{U_{0}-U(r)}} \alpha(Z, r) d r
$$

where

$$
\alpha(Z, r)=\frac{1}{4 \pi^{2} k_{0} V_{c} Z} \int_{0}^{2 \pi} \int_{0}^{2 \pi} \delta \varphi_{z}^{\prime}(Z \cos \chi, r, \psi) \cos \chi d \chi d \psi
$$

It is an extremely important fact that the non-zero $\psi$-harmonics of the field perturbation are averaged out in Eq. (11). The same holds true for any z- antisymmetric part of $\delta \varphi$. As a result, all perturbations caused by inaccuracies of positions of the central and the outer electrodes, such as shifts and tilts, affect the oscillation frequency $\omega$ only in the second or higher orders of the perturbations theory, and their contributions can be neglected. It means that the Orbitrap mass analyzer is relatively insensitive to most common assembly inaccuracies (practically up to tens of micrometers). Nevertheless, the electrode manufacturing remains a subject of serious concern.

Consider the field perturbation caused by the injection slot that represents an inevitable deviation of the outer electrode shape from the ideal equipotential surface. The electric field sags into the injection slot as illustrated in Figure 3. To simulate the effect, an imaginary axisymmetric surface was introduced to cover the injection slot, on which the potential error was calculated with the boundary element 3D algorithm as described in [7]. The zero axial harmonic of this error was considered as a boundary condition for an axisymmetric Dirichlet problem, which was solved using the finite-element method. The field perturbation $\delta \varphi(z, r)$ was averaged in accordance with Eq. (11) in the region actually occupied by the ions to obtain the function $\alpha(Z, r)$.

It is of profound importance that the field perturbation can be controlled by applying a certain voltage $V_{D}$ to the deflection electrode placed above the injection slot. Figure 4 shows the radial dependence of the coefficient $\alpha$ for different values of $V_{d}$. Though complete cancelation of the field perturbation in the entire volume of the mass analyzer is impossible, the deviation of the integral characteristic $\alpha$ can be reduced to $\sim 10^{-6}$ at certain $V_{D}=V_{D}^{*}$. In our particular case, the 'golden' ratio of voltages was $V_{D}^{*} / V_{c}=$ $423 \mathrm{~V} / 3500 \mathrm{~V} \approx 0.121$. Note that the compensating voltage $V_{D}^{*}$ generally differs from the optimal value for ion injection which is normally in the range $0-100 \mathrm{~V}$. This contradiction can be removed by switching the deflection electrode voltage to $V_{D}^{*}$ after all ions have been injected. 

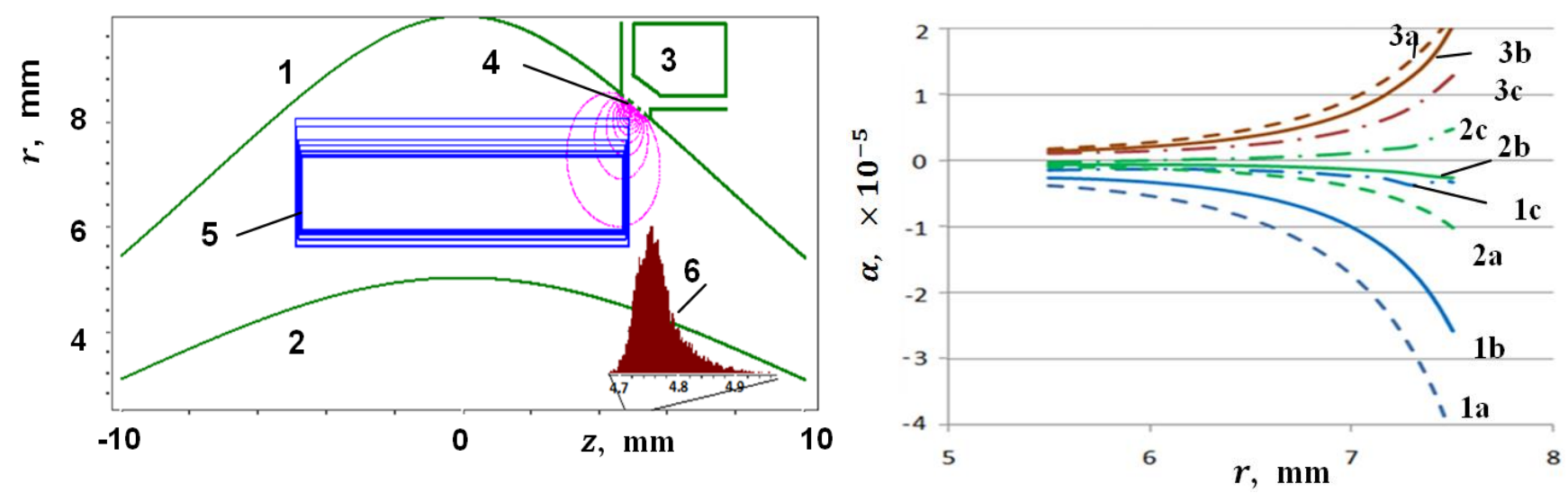

Figure 3. Scheme of the injection slot field simulation combining 3D and 2D solvers. $1-$ outer electrode, 2 - central electrode, 3 deflection electrode, 4 - imaginary surface separating the 2D simulation region, 5 populated orbits, $6-$ distribution of populated amplitudes (simulation).

Figure 4. Dimensionless coefficient $\alpha(Z, r)$ under different deflection electrode voltages $V_{D}=373 V(1 \mathrm{a}, \mathrm{b}, \mathrm{c}), V_{D}=423 \mathrm{~V}(2 \mathrm{a}, \mathrm{b}, \mathrm{c})$, and $V_{D}=473 \mathrm{~V}$ (3a,b,c). Lines with indexes ' $\mathrm{a}$ ' correspond to the section $\mathrm{Z}=4.6 \mathrm{~mm}$, ' $\mathrm{b}$ ' $-\mathrm{Z}=4.8$ $\mathrm{mm}$, and 'c' $-\mathrm{Z}=5.0 \mathrm{~mm}$ within the populated range of orbital amplitudes. $V_{c}=3500 \mathrm{~V}$

The injection slot is not the only source of the field inaccuracy. Nowadays technology allows to fabricate the electrodes with tolerances as small as one micrometer, which constitutes approximately $10^{-4}$ of the trap's dimension (compare to $10 \mathrm{~mm}$ external electrode radius). However, planning for the mass resolving powers as high as $10^{5}-10^{6}$ requires special measures to make the function Eq. (11) as flat as possible in the range of orbital parameters occupied by the injected ions. Note that the average value of $\Delta \omega$ can easily be calibrated by an appropriate choice of the central electrode voltage $V_{c}$, but its dispersion $\delta \omega^{2}=\left\langle\Delta \omega^{2}\right\rangle-\langle\Delta \omega\rangle^{2}$ is a subject to optimization. The potential penetration through the injection slot can be turned to advantage, so that a deliberate deviation of the deflection electrode voltage from $V_{D}^{*}$ compensates for the geometry imperfections. Though the field perturbation cannot be cancelled out uniformly in the entire volume of the trap, the dispersion of $\alpha(Z, r)$ can be minimized in a particular region of orbital parameters. As the populated domain of the amplitude $Z$ and the orbit radius $r$ is relatively small, a proper compensation can be achieved if both components of the gradient of $\nabla_{Z} \alpha$ and $\nabla_{r} \alpha$ vanish.

Complete control over the frequency dispersion is only possible if we have at least two independent tuning parameters which generate perturbations $\alpha$ with non-collinear gradients in the point of interest. Fortunately, the design under consideration offers a possibility of adjusting the quartz ring thickness by several micrometers in both directions. A shift of the outer electrode halves inwards or outwards induces another well-controlled smooth field perturbation as shown in Figure 5A. This perturbation, as well as the parasitic perturbations induced by the surface defects (e.g. shown in Figure 5B), can be simulated using the Bruns-Bertein method [8]. Figure 6 illustrates compensation of a parasitic perturbation by an optimal adjustment of both $V_{D}$ and the quartz ring thickness. This procedure effectively flattens out the function $\alpha$ in the populated domain of the ions' orbits. The estimate mass resolving power $R$ has increased from 80,000 (without compensation) to 300,000. 

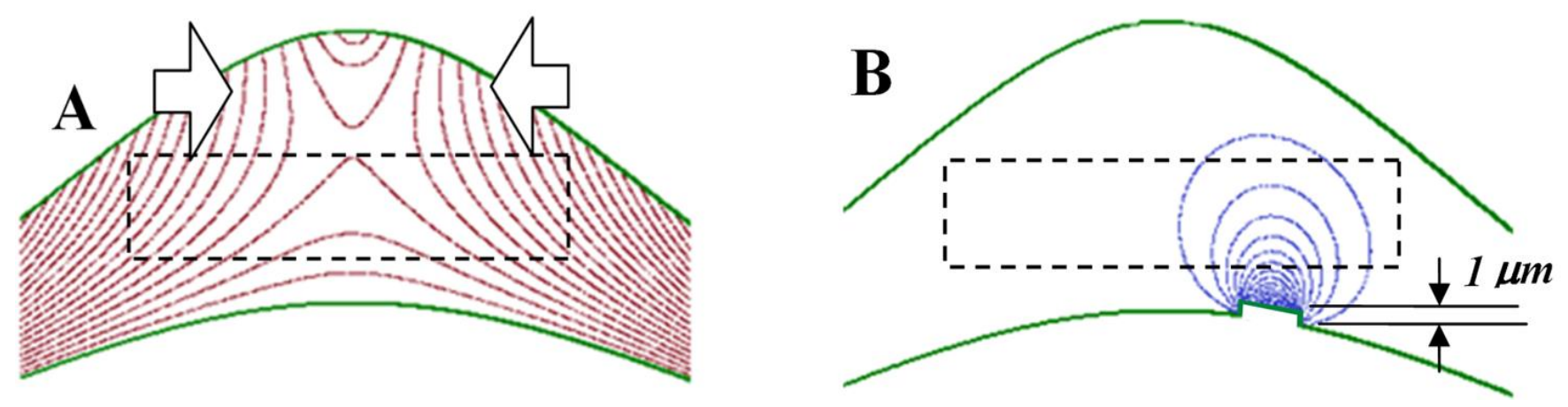

Figure 5. Electric field perturbation generated by small inward displacement of the outer electrode halves (A) and a step-shaped, $1 \mu \mathrm{m}$ high, inaccuracy of the central electrode (B).
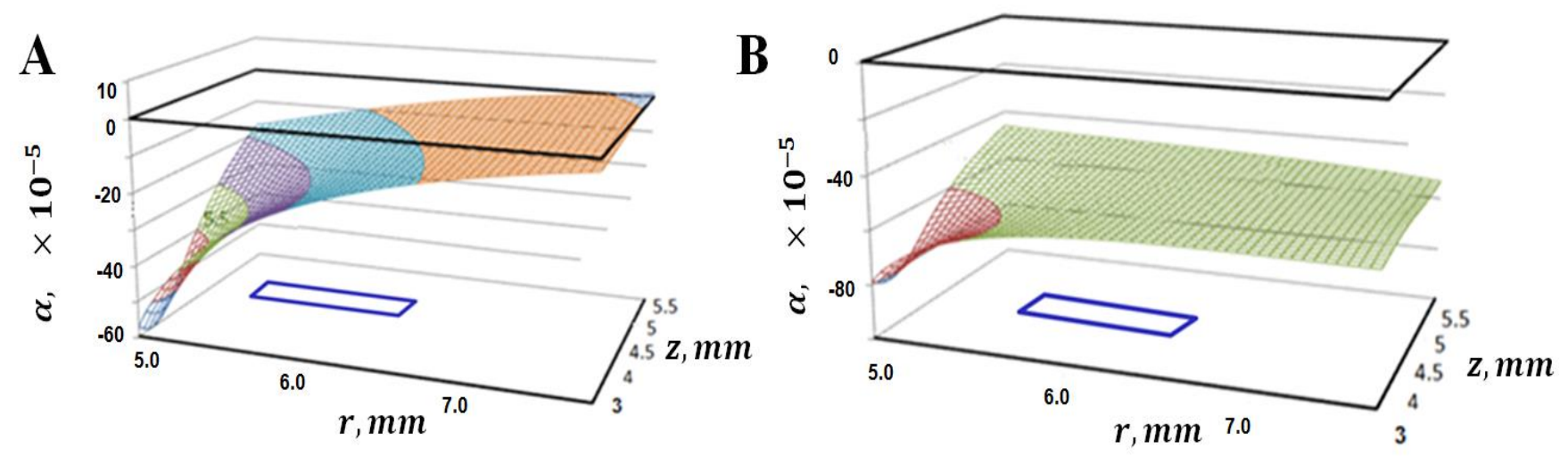

Figure 6. A - function $\alpha(Z, r)$ in case of uncorrected $1 \mu \mathrm{m}$ step perturbation as shown in Figure 5B. $\mathrm{B}$ - corrected with $-50 \mathrm{~V}$ deviation of $V_{D}$ and $8 \mu \mathrm{m}$ shift of the outer electrode halves.

Conclusions: Only two parameters, separation of the outer electrode halves and the voltage of the deflection electrode, are sufficient to effectively compensate for the perturbations of the oscillation frequency during the signal acquisition. The mass resolving power of a compensated Orbitrap mass analyzer is as high as several hundred thousand.

\section{References:}

[1] KH Kingdon, Phys. Rev. 21 (1923), p. 408.

[2] RD Knight, Appl. Phys. Lett. 38 (1981), p. 221.

[3] LN Gall et al, USSR Inventor's Certificate 1247973 (1986).

[4] A Makarov, J. Phys. D: Appl. Phys. 24 (1991), p. 533.

[5] A Makarov and E Denisov. J. Am. Soc. Mass Spectrom. 20 (2009), p. 1486.

[6] P Bolotskikh et al, Nucl. Instrum. Meth. Phys. Res. A 645 (2011), p. 146.

[7] D Greenfield and M Monastyrskiy, Adv. Imag. El. Phys. 155 (2009).

[8] F Bertein, Ann. de Radioélectricité 2 (1947), p. 379, and 3 (1948), p. 49. 